\title{
Interactive comment on "Use of an Unmanned Aircraft System to Quantify NO Emissions from a Natural Gas Boiler” by Brian Gullett et al.
}

\section{Brian Gullett et al.}

gullett.brian@epa.gov

Received and published: 10 October 2020

RC1 General Comments Gullett et al. present stack emission measurements of CO2, $\mathrm{CO}$, NO2, NO with a UAS, using a non-dispersive infrared optical absorption sensor for $\mathrm{CO} 2$ and electrochemical sensors for the other gases. They report good agreement of the measured NOx emissions with the continuous emission monitoring system (CEMS) at the test sites. Both, CEMS and the UAV measurement determine the emission via carbon balance calculations. The authors, however, do not discuss the additional value of their measurement with the UAV over the CEMS measurement. The CEMS measures the same quantity (NOx emissions) with an up to 18 times lower error (see Tab. 7 of the manuscript). The manuscript thereby does not include the description of a scientific goal or application, like e.g. the validation of the CEMS measurement, study- 
ing the dependence of the NOx emission flux on the distance to the emitting stack, or similar. The feasibility of sampling emission plumes with a UAV was shown in the cited studies of e.g. area sources or volcanoes. In many of these works the absence of a CEMS and/or chemical conversion of the measured quantities along the emission plume motivate the studies. Further, in my opinion, the scope of this journal demands a more detailed description of the 'extensive laboratory testing to verify performance and suitability' (I. 75, 76 of the manuscript) that was carried out. Especially, since electrochemical sensors are known for their rather unstable performance (see e.g. Jiao et al., 2016, https://amt.copernicus.org/articles/9/5281/2016/) and because the sensor calibration site and measurement site in this study have significantly different gas composition (plume and background air). Drifts, dependencies on environmental factors (e.g. temperature, humidity) and cross interferences with other trace gases in amounts that are typical for the sampled emission plume should be documented in the paper. Without these points thoroughly addressed I advise against publishing the manuscript in AMT.

RESPONSE to general comments: The reviewer states that the manuscript "does not include the description of a scientific goal or application" and we agree, in part, and have modified the manuscript accordingly starting with the final line in the abstract. While the advantages of a UAS platform for emission measurements are mentioned, we now explicitly stated the goal as comparison of UAS-borne emission measurements with concurrent CEM measurements (Introduction, paragraph 3). More explicit statements can lead into policy implications, for which we are unauthorized. While we are aware of previous efforts sampling volcanoes (for example), those measurements are unable to make any comments on the accuracy of the measurements as the volcano source is an unmeasured quantity. In our work we have direct measurements of the source via the in-stack CEMS, allowing us to make definitive comparisons of our ability to determine the source strength with UAS-based measurements. We agree with the reviewer that a detailed description of the sensor performance is important given their inherent potential for instability and inaccuracy. We place our potential sensors through 
rigorous in-laboratory testing covering the full range of expected target gas concentrations and then daily pre- and post-measurement calibration checks in the field. These methods are now partially mentioned in the manuscript as well as reference to manufacturers' sensor data sheets - a more extensive description of the testing is outside of the scope of this paper.

Interactive

Specific Comments

1) Faivre-Pierret et al., 1980 measured volcanic gas and particle emissions with an UAV.

RESPONSE: The current authors have previously measured gases, particles, VOCs, and SVOCs with the use of UAS-born emission equipment: [Aurell et al., 2017 - Atm Env 166]. However, questions about accuracy based upon known source strength and about the potential effects of rotor wash on emission measurements have been incompletely addressed in the literature; this paper answers some of those questions.

2) The influence of the different time constants (response times) of the sensors on the comparability of the individual measurements should be shortly discussed.

RESPONSE: Each sensor has a different response time to changes in concentration; this could be a consideration, depending on how the measurements are used. If comparisons are to be made instantaneously between pollutants, this could have a significant effect, and can be partially mitigated through use of time-averaging of concentrations. Our work took this approach in reporting concentrations, however, this was not critical, as our emission factor calculations were based upon the time-integrated sum of the concentrations over many minutes of sampling. If we had relied upon and reported instantaneous data, the longer response time of the NO2 sensor (32 $\mathrm{sec}=\mathrm{t} 95$ ) still would have had minimal effect over instantaneous emission factors as the NO2 concentration was much lower than that of $\mathrm{NO}$ and so had little effect upon the emission factor calculation. The $\mathrm{NO}$ and $\mathrm{CO} 2$ sensors have similar response times (6-9 s) and would have accurately represented the instantaneous emission factor.

Printer-friendly version

Discussion paper 
3) I.113: The underlying detection techniques of the CEMS measurements should at least be mentioned.

RESPONSE: These are now mentioned in the revised text.

4) A schematic drawing or photograph of the Kolibri setup would be illustrative.

Interactive

RESPONSE: A close up photo of the Kolibri with text explanations was inserted to comment Figure 1.

5) The CO and NO2 values in Fig. 2 should be scaled up by at least a factor of 10 in order to be clearly visible.

RESPONSE: We scaled up the NO2 and CO concentrations by 10 times.

6) In Fig. 2 it can be observed that the $\mathrm{CO} 2$ concentration reaches the upper end of the sensor's range several times. Is this always considered in the average?

RESPONSE: CO2 peaks exceeded the range of the sensor for 1,108 seconds out of the 16,500 seconds total sampling duration for the 14 -run test, or $6.7 \%$ of the sampling time. These concentrations, at their range limit, are included in the average emission factor calculations. Exclusion of the NOx and $\mathrm{C}$ data during those 1,108 seconds only affects the EFs by $3 \%$. When the sensors' ranges are exceeded, the UAS pilot is instructed to back off from the source toward a more dilute airstream.

7) I.186, 189: If the progressions of the time series as plotted in Fig. 2 were mostly determined by leaving and entering the plume with the UAV, the standard deviation of this time series would not be a good measure for the measurement error.

RESPONSE: The authors are not clear if the reviewer is referring to the RSD values cited just below Figure 2. If so, we've clarified that the cited RSDs are for flight durations and not for the observed concentrations. In any case, the reviewer is correct regarding his/her observation. Because the plume is mixing and entraining ambient air, there is considerable fluctuation in concentrations even for the stationary UAS, necessitating 
time-averaged values. While this is less of an issue for the more homogeneous gas measurements in the stack, the CEMs also use an averaged display value with a rolling AMTD average of 60 seconds which further smooths out any fluctuations in concentration.

Referee's References Jiao, W., Hagler, G., Williams, R., Sharpe, R., Brown, R., Garver, D., Judge, R., Caudill, M., Rickard, J., Davis, M., Weinstock, L., Zimmer-Dauphinee, S., and Buckley, K.: Community Air Sensor Network (CAIRSENSE) project: evaluation of low-cost sensor performance in a suburban environment in the southeastern United States, Atmos. Meas. Tech., 9, 5281-5292, https://doi.org/10.5194/amt-95281-2016, 2016. Faivre-Pierret, R., Martin, D. \& Sabroux, J.C., Contribution des Sondes Aérologiques Motorisées à l'Etude de la Physico-Chimie des Panaches Volcaniques, Bull Volcanol (1980) 43: 473. https://doi.org/10.1007/BF02597686

Authors' References: Aurell, J.; Mitchell, W.; Chirayath, V.; Jonsson, J.; Tabor, D.; Gullett, B., Field determination of multipollutant, open area combustion source emission factors with a hexacopter unmanned aerial vehicle. Atmospheric Environment 2017, 166, 433-440.

RESPONSE The authors recognize that electrochemical sensors do not have the same performance as CEMs. The referees cite Jiao et al. that tested electrochemical sensors under ambient conditions (measurement range of $\mathrm{ppb}$ ), for a minimum 30-day testing period, and without any calibrations of the sensors. Our manuscript performed calibrations just before the testing and checked the against the same calibration gases just after the test period to establish if the sensors had drifted during the testing period. For clarification we have added sensor calibration information as well as references to more extensive characterization data. The objective of conducting calibration is to ensure that the sensor measures the gas in question within acceptable levels. Calibrations of sensors and CEMs must be performed using certified gases with a known percentage of the gas tested. As such, the calibration gas mixture will always differ from the plume or stack gas mixtures. References to manufactures's technical specification sheets for each sensor have been added; these data sheets include the sensors

Interactive comment
Printer-friendly version

Discussion paper 
performance and cross sensitivity to other gases.

Comment from Referee \#1 on Referee \#2 comments, RC3

AMTD

Agreed [with Referee \#1], but suggest this can be addressed by brief reference to other publications by the authors (if available) or through the use of supplemental material and data (i.e., inclusion in body of paper will distract from focus of research).

Interactive

comment

RESPONSE: The authors assume that Referee \#1 agrees to Referee \#2 comment about "extensive testing" as described in RC2 below. We have added this in the text.

Interactive comment on Atmos. Meas. Tech. Discuss., doi:10.5194/amt-2020-108, 2020. 